\title{
AN INTERIOR-POINT METHOD FOR LINEAR OPTIMIZATION BASED ON A TRIGONOMETRIC KERNEL FUNCTION
}

\author{
ZAHRA MOABERFARD ${ }^{1,3}$, SAJAD FATHI-HAFSHEJANI $^{2, *}$, ALIREZA FAKHARZADEH J. $^{2,3}$ \\ ${ }^{1}$ Department of Computer Engineering, Apadana Institute of Higher Education, Shiraz, Iran \\ ${ }^{2}$ Department of Mathematics, Shiraz University of Technology, P.O. Box 71555-313, Shiraz, Iran \\ ${ }^{3}$ Fars Elites Foundation, P.O. Box 71966-98893, Shiraz, Iran
}

\begin{abstract}
In this paper, we propose a large-update primal-dual interior point algorithm for linear optimization problems based on a new kernel function with a trigonometric barrier term. By means of some simple analysis tools, we prove that the interiorpoint algorithm based on the new proposed kernel function meets $O\left(\sqrt{n} \log n \log \frac{n}{\varepsilon}\right)$ as the worst-case complexity bound for the large-update method, which coincides with the best-known complexity result for large-update method. Finally, some numerical results of performing the algorithm are presented.
\end{abstract}

Keywords. Trigonometric kernel function; Linear optimization; Interior-point method; Large-update methods.

2010 Mathematics Subject Classification. 90C51, 90C05.

\section{INTRODUCTION}

In this paper, we deal with primal-dual Interior-Point Method (IPM) for solving the standard Linear Optimization (LO) problem:

$$
\min \left\{c^{T} x: A x=b, x \geq 0\right\}
$$

and its dual as:

$$
\max \left\{b^{T} y: A^{T} y+s=c, s \geq 0\right\}
$$

where $A \in \mathbb{R}^{m \times n}, x, c, s \in \mathbb{R}^{n}$ and $y, b \in \mathbb{R}^{m}$. Moreover, we assume, throughout the paper, that the matrix $A$ has full row rank, i.e., $\operatorname{rank}(A)=m \leq n$ and problems (P) and (D) satisfy the Interior Point Condition (IPC), i.e., there exists a strictly feasible point, namely, $x^{0}>0$ and $\left(y^{0}, s^{0}\right)$ with $s^{0}>0$, such that

$$
\begin{aligned}
A x^{0} & =b, & x^{0}>0, \\
A^{T} y^{0}+s^{0} & =c, & s^{0}>0 .
\end{aligned}
$$

${ }^{*}$ Corresponding author.

E-mail addresses: zahra.moaberfard@gmail.com (Z. Moaberfard), s.fathi@ sutech.ac.ir (S. Fathi-Hafshejani), a_fakharzadeh @ sutech.ac.ir (A. Fakharzadeh J.).

Received August 31, 2019; Accepted November 24, 2019.

(C)2019 Journal of Nonlinear Functional Analysis 
In the last decade, powerful mathematical approaches have been proposed for solving convex optimization problems. For example, we refer to the IPM that is one of the methods that has received a lot of attention by the researchers. An effective work in this direction was first done by by Karmarkar in [1]. Then, Kojima, Mizuno and Yoshise[2] and Megiddo [3] introduced the concept of primal-dual IPMs. The IPMs from LO was first developed to other convex optimization problems such as Convex Quadratic Optimization (CQO), Semidefinite Optimization (SDO), Second Order Cone Optimization (SOCO), and Nonlinear Complementarity Problem (NCP) by Nesterov and Nemirovskii in [4].

Peng, Roos and Terlaky in [5] introduced the concept of kernel-based interior point methods for LO problems. Kernel functions play an important role in the design and analysis of primal-dual IPMs. In fact, they were used to determine the search directions as well as were applied to measure the distance between the given iterate and the $\mu$-center. They illustrated that their algorithm based on the so-called Self-Regular (SR) kernel functions for LO problems has the best known iteration complexity bounds for large and small-update methods, namely, $O\left(\sqrt{n} \log n \log \frac{n}{\varepsilon}\right)$ and $O\left(\sqrt{n} \log \frac{n}{\varepsilon}\right)$, respectively. Since then, several attempts for introducing non-SR kernel functions in order to at least meeting the complexity results of SR barrier functions have been started. For comparative studies on the kernel functions for LO, we refer to $[6,7,8,9,10,11]$ and the references therein.

El Ghami et al. in [12] introduced a new kernel function with trigonometric barrier term and showed an interior point algorithm for LO problems based on the trigonometric kernel function enjoys $O\left(n^{\frac{3}{4}} \log \frac{n}{\varepsilon}\right)$ worst case iteration bound. Later on, Kheirfam in [13] proposed a new kernel function with trigonometric barrier term for SDO and obtained the same complexity obtained by El Ghami et al. in [12]. Peyghami, Fathi-Hafshejani and Shirvani in [14] introduced a new kernel function with a trigonometric barrier term and proved that the iteration complexity of large update IPMs for LO problems based on this kernel function meets $O\left(n^{\frac{2}{3}} \log \frac{n}{\varepsilon}\right)$. This bound significantly improved the result obtained by El Ghami et al. $[12,15]$ and Khierfam [13] for large-update methods. Bouafia et al. in [16] designed a new IPM based on the trigonometric kernel function for LO problems. They proved that their algorithm based on the new kernel function has the best complexity bound for large-update methods. Another recent attempts in this field can be found in $[13,15,17,18,19,20,21,22,23,24,25,26]$. Some of these functions obtained the so far best known iteration complexity, see, e.g., [16, 18, 19, 20, 21, 22, 23, 24, 25, 26]. Table 1 reveals some other of existing trigonometric kernel functions as well as their complexity.

It has to be noted that, due to [6], all the researches in introducing new kernel functions are basically focused on finding a kernel function for which the complexity of large-update methods is equal to (or even better than) $O\left(\sqrt{n} \log \frac{n}{\varepsilon}\right)$, or show that such a kernel function does not exist. This motivates us to work on theoretical complexity aspects of several kernel functions which are not self-regular. The research line of this paper coincides to this fact. Indeed, our main concern is on deriving the theoretical complexity of a new proposed kernel function in the way of verifying the aforementioned question.

Motivate by these results, a new kernel function with a trigonometric barrier term is introduced. By means of some simple analysis tools, we analyze the large-update primal dual IPM based on the new proposed kernel function and show that the algorithm enjoys $O\left(\sqrt{n} \log n \log \frac{n}{\varepsilon}\right)$ as the worst case iteration complexity bound. We also present some numerical results. The results are obtained by performing the interior point algorithm based on the new proposed kernel function and six kernel functions defined in literature. Comparison of the obtained results shows that the new proposed kernel function outperforms the other considered kernel functions. 
TABLE 1. Considered kernel functions.

\begin{tabular}{lllll}
\hline$i$ & Kernel function $\psi_{i}$ & large-update & Years & Ref. \\
\hline 1 & $\frac{t^{2}-1}{2}+\frac{6}{\pi} \tan \left(\frac{1-t}{2+4 t} \pi\right)$ & - & 2004 & {$[6]$} \\
\hline 2 & $\frac{t^{2}-1}{2}+\frac{6}{\pi} \tan \left(\frac{1-t}{2+4 t} \pi\right)$ & $O\left(n^{\frac{3}{4}} \log \frac{n}{\varepsilon}\right)$ & 2012 & {$[12]$} \\
\hline 3 & $\frac{t^{2}-1}{2}-\log (t)+\frac{1}{8} \tan ^{2}\left(\frac{1-t}{2+4 t} \pi\right)$ & $O\left(n^{\frac{2}{3}} \log \frac{n}{\varepsilon}\right)$ & 2014 & {$[14]$} \\
\hline 4 & $\frac{t^{2}-1}{2}-\int_{1}^{t} e^{\left(3\left(\tan \left(h\left(\frac{\pi}{2+2 x}\right)-1\right)\right)\right.} d x$ & $O\left(\sqrt{n} \log ^{2} n \log \frac{n}{\varepsilon}\right)$ & 2014 & {$[27]$} \\
\hline 5 & $\frac{t^{2}-1}{2}-\log (t)+\lambda \tan ^{2}(h(t))$ & $O\left(n^{\frac{2}{3}} \log \frac{n}{\varepsilon}\right)$ & 2014 & {$[28]$} \\
& $h(t):=\frac{\pi(1-t)}{3 t+2}, 0<\lambda \leq \frac{8}{25}$ & & & \\
\hline 6 & $t^{2}-2 t+\frac{1}{\sin \left(\frac{\pi t}{1+t}\right)}$ & $O\left(n^{\frac{3}{4}} \log \frac{n}{\varepsilon}\right)$ & 2015 & {$[29]$} \\
\hline 7 & $\frac{(t-1)^{2}}{2}+\frac{(t-1)^{2}}{2 t}+\frac{1}{8} \tan ^{2}\left(\frac{1-t}{2+4 t} \pi\right)$ & $O\left(n^{\frac{2}{3}} \log \frac{n}{\varepsilon}\right)$ & 2015 & {$[30]$} \\
\hline 8 & $\frac{t^{2}-1}{2}+\frac{4}{p \pi}\left(\tan ^{p}\left(\frac{\pi}{2+2 x}\right)-1\right), p \geq 2$ & $O\left(p n^{\frac{p+2}{2(p+1)}} \log \frac{n}{\varepsilon}\right)$ & 2016 & {$[16]$} \\
\hline \hline
\end{tabular}

The paper is organized as follows: In Section 2, we recall some concepts of IPMs LO problems. In Section 3, we introduce the new kernel function and some of its properties. Section 4 is devoted to describe the proximity reduction during an inner iteration. We also obtain a default value for the step size in this section. The worst case iteration bound for the large update primal-dual IPMs based on the new kernel function is provided in Section 5. In Section 6, we present some numerical results. Finally, some concluding remarks are given in Section 7.

We use the following notational conventions: Throughout the paper, we use $\|$.$\| to denote the Euclidian$ norm of a vector. The nonnegative and positive orthants are denoted by $\mathbb{R}_{+}^{n}$ and $\mathbb{R}_{++}^{n}$, respectively. For a vector $x=\left(x_{1}, \ldots, x_{n}\right) \in \mathbb{R}^{n}, x_{*}$ is the minimum component of $x$. For given vectors $x$ and $s$, the vectors $x s$ and $\frac{x}{s}$ denote the coordinate-wise operations on the vectors, i.e., whose components are $x_{i} s_{i}$ and $\frac{x_{i}}{s_{i}}$, respectively. We say that $f(t)=\Theta(g(t))$ if there exist positive constants $\omega_{1}$ and $\omega_{2}$ so that $\omega_{1} g(t) \leq f(t) \leq \omega_{2} g(t)$, for all $t \in \mathbb{R}_{++}$. We also say that $f(t)=O(g(t))$ if there exists a positive constant $\omega$ so that $f(t) \leq \omega g(t)$, for all $t \in \mathbb{R}_{++}$.

\section{THE NEW KERNEL FUNCTION}

This section introduces a new kernel function with trigonometric barrier term and reveals its properties. Let us define the function $\psi(t)$ as:

$$
\psi(t)=p \frac{t^{2}-1}{2}+\frac{4}{\pi}(\exp [p(\tan (h(t))-1)]-1), \quad p \geq 1,
$$

where

$$
h(t)=\frac{\pi}{2+2 t} .
$$

To investigate the behavior of the new kernel function around the minimum point, the diagram of the function $\psi(t)$ is plotted for three different successive values of parameter $p=\{1,1.5,2\}$ in Fig. 1

Now, we prove that the $\psi(t)$ is a kernel function. To this end, one can easily see that $\lim _{t \rightarrow 0^{+}} h(t)=$ $\frac{\pi}{2}$, therefore $\lim _{t \rightarrow 0^{+}} \psi(t) \rightarrow+\infty$. On the other hand, $\lim _{t \rightarrow+\infty} h(t)=0$, which in turn implies that $\lim _{t \rightarrow+\infty} \psi(t) \rightarrow+\infty$. All these relations say that the $\psi(t)$ defined by (2.1) is a kernel function [6]. 


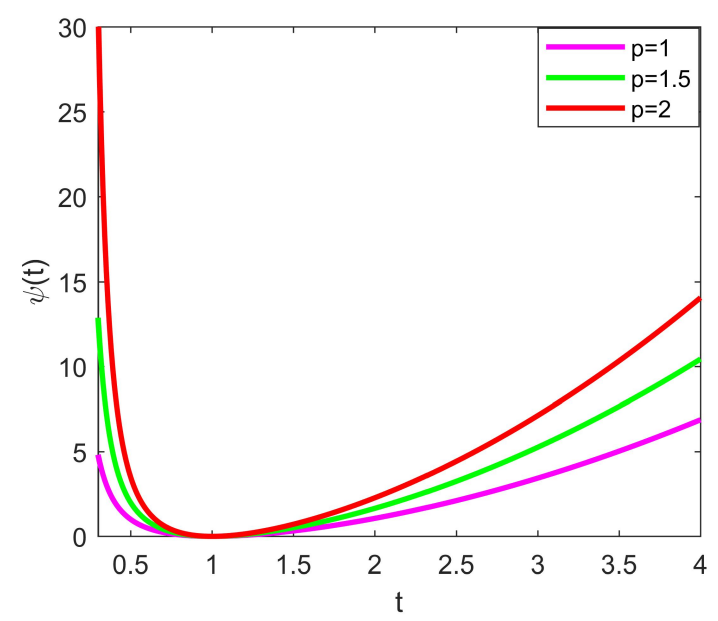

FIGURE 1. The behavior of the new kernel function for $p=\{1,1.5,2\}$.

In the following, we present the first three derivatives of the proposed kernel function which are given by

$$
\begin{aligned}
\psi^{\prime}(t) & =p t-\frac{8 p}{(2+2 t)^{2}}\left(1+\tan ^{2}(h(t))\right) \exp [p(\tan (h(t))-1)] \\
\psi^{\prime \prime}(t) & =p+\frac{2 p \varpi(t)}{(1+t)^{3}}\left[2+\frac{\pi}{1+t} \tan (h(t))+\frac{\pi p}{2+2 t} \varpi(t)\right] K(t) \\
\psi^{\prime \prime \prime}(t) & =-\frac{32 p \varpi(t)}{(2+2 t)^{4}}\left[3+\frac{11 \tan (h(t))}{2+2 t}+\varpi(t)\left(\frac{8 \pi p t+8 \pi p+2 \pi^{2}}{(2+2 t)^{2}}\right)+\frac{4 \pi}{(2+2 t)^{2}} M(t)\right] K(t)
\end{aligned}
$$

where

$$
\begin{aligned}
M(t) & :=\left(\pi \tan ^{2}(h(t))+p^{2} \tan (h(t)) \varpi(t)+p^{2} \pi \varpi^{2}(t)\right), \\
K(t) & :=\exp [p(\tan (h(t))-1)], \\
\varpi(t) & :=1+\tan ^{2}(h(t)) .
\end{aligned}
$$

The next lemma is on a lower bound for the function $\tan (h(t))$ for all $t \in(0,1]$. The proof can be found in [27].

Lemma 2.1. For the function $h(t)$ given by (2.2), one has

$$
\tan (h(t))-\frac{1}{\pi t}>0, \quad 0<t \leq 1
$$

Our next lemma reveals some key properties of the new kernel function.

Lemma 2.2. For the kernel function defined by (2.1), we have

i): $\psi^{\prime \prime}(t)>1, \quad \forall t>0$,

ii): $t \psi^{\prime \prime}(t)-\psi^{\prime}(t)>0, \quad \forall t>1$,

iii): $t \psi^{\prime \prime}(t)+\psi^{\prime}(t)>0, \quad \forall t>0$,

iv): $\psi^{\prime \prime \prime}(t)<0, \quad \forall t>0$. 
Proof. Given the structure of the function $h(t)$ defined by (2.2), one can easily see that

$$
\begin{aligned}
& \tan (h(t)) \geq 1, \quad \text { for all } t \in(0,1], \\
& \tan (h(t)) \in(0,1), \quad \text { for all } t>1, \\
& \varpi(t) \text { and } K(t) \geq 0, \quad \text { for all } t>0 .
\end{aligned}
$$

The first item is easily obtained by using the following relation

$$
\psi^{\prime \prime}(t)=p+\frac{2 p \varpi(t)}{(1+t)^{3}}\left[2+\frac{\pi}{1+t} \tan (h(t))+\frac{\pi p}{2+2 t} \varpi(t)\right] K(t) \geq p \geq 1 .
$$

The second item is proved as

$$
t \psi^{\prime \prime}(t)-\psi^{\prime}(t)=\frac{2 p \varpi(t)}{(1+t)^{3}}\left[3 t+1+\frac{t \pi}{1+t} \tan (h(t))+\frac{t \pi p}{2+2 t} \varpi(t)\right] K(t)>0 .
$$

To prove (iii), we consider two different states. First, we assume that $t \in(0,1]$. By using Lemma 2.1, and the fact that $\tan (h(t)) \geq 1$, we conclude that

$$
\begin{aligned}
t \psi^{\prime \prime}(t)+\psi^{\prime}(t) & =2 p t+\frac{2 p \varpi(t)}{(1+t)^{3}}\left[2 t+\frac{\pi t}{1+t} \tan (h(t))+\frac{\pi p t}{2+2 t} \varpi(t)-(1+t)\right] K(t) \\
& \geq 2 p t+\frac{2 p \varpi(t)}{(1+t)^{3}}\left[t+\frac{1}{1+t}+\frac{\pi p t}{2+2 t} \varpi(t)-1\right] K(t) \\
& =2 p t+\frac{2 p \varpi(t)}{(1+t)^{3}}\left[\frac{t^{2}}{1+t}+\frac{\pi p t}{2+2 t} \varpi(t)\right] K(t)>0,
\end{aligned}
$$

where the inequality is obtained by using lemma 2.1. To prove the second case, that is, $t>1$, we use the fact that $\psi^{\prime}(1)=0$ and $\psi^{\prime \prime}(t)>1$. These relations imply that the function $\psi^{\prime}(t)$ is an increasing and non-negative function, for all $t>0$, which points out $t \psi^{\prime \prime}(t)+\psi^{\prime}(t)>0$. To prove (iv), we use the fact that $\varpi(t), K(t)$ and $\tan (h(t))$ have non-negative values for all $t>0$. Therefore, we have

$$
\psi^{\prime \prime \prime}(t)=-\frac{32 p \varpi(t)}{(2+2 t)^{4}}\left[3+\frac{11 \tan (h(t))}{2+2 t}+\varpi(t)\left(\frac{8 \pi p t+8 \pi p+2 \pi^{2}}{(2+2 t)^{2}}\right)+\frac{4 \pi}{(2+2 t)^{2}} M(t)\right] K(t)<0,
$$

which completes the proof of the lemma.

Now, we reveal the exponential convexity (e-convexity) property of the kernel function that plays an important role in the complexity analysis of the primal-dual IPMs based on the kernel function. Three equivalent statements for the e-convexity property of a kernel function are illustrated by the following technical lemma.

Lemma 2.3. [10] Suppose that $\psi(t)$, for $t>0$, is a twice continuously differentiable function. Then, the following statements are equivalent:

i): $\psi\left(\sqrt{t_{1} t_{2}}\right) \leq \frac{1}{2}\left(\psi\left(t_{1}\right)+\psi\left(t_{2}\right)\right), \quad$ for $t_{1}, t_{2}>0$,

ii): $\psi^{\prime}(t)+t \psi^{\prime \prime}(t) \geq 0 \quad$ for $t>0$,

iii): $\psi\left(e^{\xi}\right)$ is a convex function.

Lemma 2.3 along with the third part of Lemma 2.2 reveals that the new proposed kernel function defined as (2.1) has the e-convexity property. 
In the following, we examine another property of the kernel function that is calculated by using $\psi(1)=$ $\psi^{\prime}(1)=0$. This property says that the kernel function $\psi(t)$ can be described by its second derivative according to

$$
\psi(t)=\int_{1}^{t} \int_{1}^{\xi} \psi^{\prime \prime}(\zeta) d \zeta d \xi
$$

Lemma 2.4. Let $\psi(t)$ be the new kernel function defined by (4), and $t>0$. Thus,

$$
\frac{p}{2}(t-1)^{2} \leq \psi(t) \leq \frac{1}{2 p} \psi^{\prime}(t)^{2} .
$$

Proof. The relation (2.9) along with the property $\psi^{\prime \prime}(t) \geq p$ for all $t>0$ implies that

$$
\psi(t)=\int_{1}^{t} \int_{1}^{\xi} \psi^{\prime \prime}(\zeta) d \zeta d \xi \geq \int_{1}^{t} \int_{1}^{\xi} p d \zeta d \xi=\frac{p}{2}(t-1)^{2}
$$

To prove the second inequality, we have

$$
\begin{aligned}
\psi(t) & =\int_{1}^{t} \int_{1}^{\xi} \psi^{\prime \prime}(\zeta) d \zeta d \xi \leq \frac{1}{p} \int_{1}^{t} \int_{1}^{\xi} \psi^{\prime \prime}(\zeta) \psi^{\prime \prime}(\xi) d \zeta d \xi \\
& =\frac{1}{p} \int_{1}^{t} \psi^{\prime \prime}(\xi) \psi^{\prime}(\xi) d \xi=\frac{1}{p} \int_{1}^{t} \psi^{\prime}(\xi) d \psi^{\prime}(\xi)=\frac{1}{2 p}\left(\psi^{\prime}(t)\right)^{2}
\end{aligned}
$$

Now, we define the norm-based proximity measure $\delta(v)$ as below

$$
\delta(v):=\frac{1}{2}\|\nabla \Psi(v)\|=\frac{1}{2} \sqrt{\sum_{i=1}^{n}\left(\psi^{\prime}\left(v_{i}\right)\right)^{2}}, \quad v \in R_{++}^{n} .
$$

The next lemma describes relation between the proximity measure and norm based proximity measure.

Lemma 2.5. For the new kernel function $\psi(t)$ defined by (4), we have

i): $p \Psi(v) \leq 2 \delta(v)^{2}$,

ii): $\|v\| \leq \sqrt{n}+\sqrt{\frac{2}{p} \Psi(v)} \leq \sqrt{n}+2 \delta(v)$.

Proof. Lemma 2.4 and (2.10) imply that the first item of the lemma. The second item is obtained by using Lemma 2.4, the Cauchy-Schwarz inequality and the first item

$$
\Psi(v)=\sum_{i=1}^{n} \psi\left(v_{i}\right) \geq \frac{p}{2} \sum_{i=1}^{n}\left(v_{i}-1\right)^{2}=\frac{p}{2}\left(\|v\|^{2}-2 e^{T} v+n\right) \geq \frac{p}{2}(\|v\|-\|e\|)^{2},
$$

which implies that

$$
\|v\| \leq\|e\|+\sqrt{\frac{2}{p} \Psi(v)}=\sqrt{n}+\sqrt{\frac{2}{p} \Psi(v)} .
$$

Thus,

$$
\|v\| \leq \sqrt{n}+2 \delta(v)
$$

which completes the proof.

As a result of the second part of Lemma 2.5, we have the following. 
Corollary 2.6. If $\Psi(v) \geq 1$, then

$$
\delta:=\delta(v) \geq \sqrt{\frac{p}{2}} \geq \sqrt{\frac{1}{2}} .
$$

In the sequel, we investigate the growth behavior of the new kernel function and its related real value matrix function.

Lemma 2.7. Assume that $\beta \geq 1$ and the function $\psi(t)$ is given by (2.1). Then,

$$
\psi(\beta t) \leq \psi(t)+\frac{p}{2}\left(\beta^{2}-1\right) t^{2} .
$$

Proof. The proof is similar to the proof of Lemma 4.1 in [27]. Therefore, we omit it here.

Lemma 2.8. Let $0<\theta<1$ and $v_{+}=\frac{v}{\sqrt{1-\theta}}$. Then

$$
\Psi\left(v_{+}\right) \leq \Psi(v)+\frac{p \theta}{2(1-\theta)}\left(\frac{2}{p} \Psi(v)+2 \sqrt{\frac{2 n}{p} \Psi(v)}+n\right) .
$$

Proof. Lemma 2.7 with $\beta=\frac{1}{\sqrt{1-\theta}}$ implies that

$$
\begin{aligned}
\Psi\left(v_{+}\right) & =\Psi(\beta v)=\sum_{i=1}^{n} \psi\left(\beta v_{i}\right) \\
& \leq \sum_{i=1}^{n}\left(\psi\left(v_{i}\right)+\frac{1}{2}\left(\beta^{2}-1\right) v_{i}^{2}\right) \\
& =\Psi(v)+\frac{p}{2} \sum_{i=1}^{n}\left(\beta^{2}-1\right) v_{i}^{2} \\
& =\Psi(v)+\frac{p \theta\|v\|^{2}}{2(1-\theta)} .
\end{aligned}
$$

By using the second part of Lemma 2.5, we have

$$
\Psi\left(v_{+}\right) \leq \Psi(v)+\frac{p \theta}{2(1-\theta)}\left(\frac{2}{p} \Psi(v)+2 \sqrt{\frac{2 n}{p} \Psi(v)}+n\right) .
$$

\section{The Central Path}

The Karush-Kuhn-Tucker (KKT) conditions for both problems (P) and (D) can be considered as:

$$
\begin{array}{rlrl}
A x & =b, & & x \geq 0 ; \\
A^{T} y+s & =c, & & s \geq 0 ; \\
x s & =0 . &
\end{array}
$$

By replacing the third equation in (3.1), with the nonlinear parametric equation $x s=\mu \mathbf{e}$, in which $\mu$ is a real positive parameter and $\mathbf{e}=(1,1, \ldots, 1)^{T}$, we have the following system

$$
\begin{array}{rlr}
A x & =b, & x \geq 0 ; \\
A^{T} y+s & =c, \quad s \geq 0 ; \\
x s & =\mu \mathbf{e} .
\end{array}
$$


By considering Assumptions $A 1$ and $A 2$, a unique solution as $(x(\mu), y(\mu), s(\mu))$ of system (3.2) for $\mu>0$ can be obtained [10]. We call $x(\mu)$ and $(y(\mu), s(\mu))$ the $\mu$-centers of (P) and (D), respectively. The set of $\mu$-centers, for all $\mu>0$, defines a homotopy path which is called the central path of (3.2) [31]. As $\mu \rightarrow 0$, the limit of the central path exists and converges to the analytic center of the optimal solution set of (P) and (D), see e.g. [3, 32].

The next aim is to calculate the search direction $(\Delta x, \Delta y, \Delta s)$. In this approach, the following system is solved

$$
\begin{aligned}
A \Delta x & =0 \\
A^{T} \Delta y+\Delta s & =0 \\
x \Delta s+s \Delta x & =\mu \mathbf{e}-x s .
\end{aligned}
$$

The scaled vector $v$ is defined as

$$
v:=\sqrt{\frac{x s}{\mu}}
$$

We define the new search directions $d_{x}$ and $d_{s}$

$$
d_{x}=\frac{v \Delta x}{x}, \quad d_{s}=\frac{v \Delta s}{s} .
$$

Now, we able to calculus the search direction $\left(d_{x}, \Delta y, d_{s}\right)$ by solving the following system

$$
\begin{aligned}
\bar{A} d_{x} & =0 ; \\
\bar{A}^{T} \Delta y+d_{s} & =0 ; \\
d_{x}+d_{s} & =v^{-1}-v
\end{aligned}
$$

where

$$
\begin{aligned}
\bar{A} & :=\frac{1}{\mu} A V^{-1} X=A S^{-1} V \\
V & :=\operatorname{diag}(v), X:=\operatorname{diag}(x), S:=\operatorname{diag}(s) .
\end{aligned}
$$

As mentioned in [10], system (3.5) has a unique solution and search direction $(\Delta x, \Delta y, \Delta s)$ can be easily obtained by applying (3.4). Now, we replace the right-hand side of the last equation in (3.5) by the negative gradient of the proximity function $\Psi(v)=\sum_{i=1}^{n} \psi\left(v_{i}\right)$, in which $\psi(t)$ is the new proposed kernel function given by (1). Therefore, system (3.5) can be rewritten as

$$
\begin{aligned}
\bar{A} d_{x} & =0 ; \\
\bar{A}^{T} \Delta y+d_{s} & =0 ; \\
d_{x}+d_{s} & =-\nabla \Psi(v) .
\end{aligned}
$$

In [10], it was showed that this system has a unique solution. From the discussion above, we conclude that

$$
d_{x}=d_{s}=0 \Leftrightarrow \psi^{\prime}(v)=0 \Leftrightarrow v=\mathbf{e} \Leftrightarrow \Psi(v)=0,
$$

namely, if and only if $x s=\mu \mathbf{e}$, i.e., $x=x(\mu)$ and $s=s(\mu)$. Otherwise, we have $\Psi(v)>0$. Hence, if $(x, y, s) \neq(x(\mu), y(\mu), s(\mu))$, then $(\Delta x, \Delta y, \Delta s) \neq 0$, which implies that we can compute step size $\alpha$ by using some line search rules to obtain a new triple $\left(x_{+}, y_{+}, s_{+}\right)$as below

$$
x_{+}=x+\alpha \Delta x, \quad y_{+}=y+\alpha \Delta y, \quad s_{+}=s+\alpha \Delta s .
$$


Summarizing the above argument, we can outline this procedure in the following primal-dual interior point scheme [10].

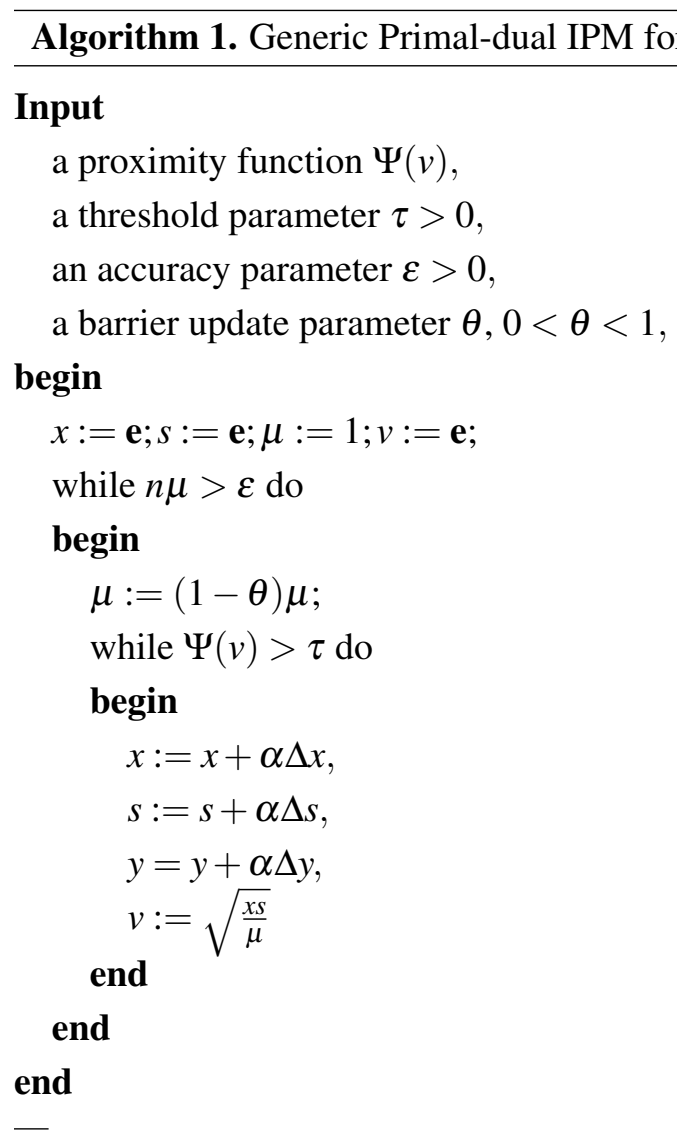

Now, we illustrate an iteration of Algorithm 1. Starting a strictly feasible point $\left(x^{0}, y^{0}, s^{0}\right)$, and assume that, for given $\mu>0$, an approximation of the $\mu$-center $(x(\mu), y(\mu), s(\mu))$ is at hand. If $n \mu \leq \varepsilon$, then the algorithm is terminated. Otherwise, the parameter $\mu$ is decreased by the factor $1-\theta$, where $\theta \in(0,1)$. This part of the algorithm is known as outer iteration loop. While the value of $\Psi(v)$ is greater than the threshold $\tau$, the new iterate is computed by taking Newton steps. In fact, this part of the algorithm constitutes the inner iterations loop. This procedure is repeated until we get to the point in which $n \mu \leq \varepsilon$. We note that the total number of iterations is given by multiplication of the inner and outer iterations.

\section{AN ESTIMATION FOR THE STEP SIZE}

The goal of this section is to calculate a lower bound for the step size during an inner iteration of Algorithm 1. Let us present the new point after an inner iteration as:

$$
x_{+}=x+\alpha \Delta x, \quad y_{+}=y+\alpha \Delta y, \quad s_{+}=s+\alpha \Delta s,
$$

where $\alpha$ is the so called the step size. According to (3.4), we can present the new point as

$$
x_{+}=\frac{x}{v}\left(v+\alpha d_{x}\right), \quad y_{+}=y+\alpha \Delta y, \quad s_{+}=\frac{s}{v}\left(v+\alpha d_{s}\right) .
$$


Define $v_{+}:=\sqrt{\frac{x_{+} s_{+}}{\mu}}$ implies that

$$
v_{+}^{2}=\frac{x_{+} s_{+}}{\mu}=\left(v+\alpha d_{x}\right)\left(v+\alpha d_{s}\right) .
$$

By using the $e$-convexity property, the following results is obtained

$$
\Psi\left(v_{+}\right)=\Psi\left(\sqrt{\left(v+\alpha d_{x}\right)\left(v+\alpha d_{s}\right)} \leq \frac{1}{2}\left(\Psi\left(v+\alpha d_{x}\right)+\Psi\left(v+\alpha d_{s}\right)\right) .\right.
$$

The gap between the proximity function before and after one step below is defined as a function with respect $\alpha$

$$
f(\alpha):=\Psi\left(v_{+}\right)-\Psi(v)
$$

Now, similar to [6], the default step size $\alpha$ is defined as

$$
\tilde{\alpha}=\frac{1}{\psi^{\prime \prime}(\rho(2 \delta))},
$$

where $\rho:[0, \infty) \rightarrow(0,1]$ is the inverse of the function $-\frac{1}{2} \psi^{\prime}(t)$ in the interval $(0,1]$.

Duo to [6], an upper bound for function $f(\alpha)$ is calculated by using the following lemma.

Lemma 4.1. [6] For any $\alpha$ satisfying $\alpha \leq \bar{\alpha}$, one has

$$
f(\alpha) \leq-\alpha \delta^{2}
$$

Lemma 4.2. Assume that $\Psi(v) \geq 1, \rho$ is the inverse of the function $-\frac{1}{2} \psi^{\prime}(t)$ in the interval $(0,1]$ and $\tilde{\alpha}$ is defined by (4.3). Thus,

$$
f(\tilde{\alpha}) \leq-\frac{\delta^{2}}{\psi^{\prime \prime}(\rho(2 \delta))} \leq \Theta\left(-\frac{\delta}{\log ^{2}(8 \delta+2)}\right)
$$

Proof. Lemma 4.1 along with $\tilde{\alpha} \leq \bar{\alpha}$ implies that $f(\tilde{\alpha}) \leq-\tilde{\alpha} \delta^{2}$. Now, we compute the inverse function $-\frac{1}{2} \psi^{\prime}(t)$, for $0<t \leq 1$. By using $-\frac{1}{2} \psi^{\prime}(t)=s$, we can calculate $t$ as a function of $s$. To this end, we have

$$
-\left(p t-\frac{8 p}{(2+2 t)^{2}}\left(1+\tan ^{2}(h(t))\right) \exp [p(\tan (h(t))-1)]\right)=2 s,
$$

which implies that $p\left(1+\tan ^{2}(h(t))\right) \exp [p(\tan (h(t))-1)] \leq 2(2 s+p)$, in which the last inequality is obtained from the fact that $t \in(0,1]$. Now, letting $t=\rho(2 \delta)$, we have $4 \delta=-\psi^{\prime}(t)$, which implies that

$$
\left(1+\tan ^{2}(h(t))\right) \exp [p(\tan (h(t))-1)] \leq 8 \delta+2
$$

Using the fact that $\exp [p(\tan (h(t))-1)] \leq\left(1+\tan ^{2}(h(t))\right) \exp [p(\tan (h(t))-1)]$, we conclude that

$$
\begin{aligned}
& \exp [p(\tan (h(t))-1)] \leq 8 \delta+2 \\
& p \tan (h(t)) \leq \log (8 \delta+2) \\
& \tan (h(t)) \leq \frac{1}{p} \log (8 \delta+2)
\end{aligned}
$$


Now, using (4.6) and (4.7), and the fact that $t \in(0,1]$, we obtain the following relations

$$
\begin{aligned}
\psi^{\prime \prime}(t) & =p+\frac{2 p \varpi(t)}{(1+t)^{3}}\left[2+\frac{\pi}{1+t} \tan (h(t))+\frac{\pi p}{2+2 t} \varpi(t)\right] K(t) \\
& =p+p \varpi(t) K(t) \frac{2}{(1+t)^{3}}\left[2+\frac{\pi}{1+t} \tan (h(t))+\frac{\pi p}{2+2 t} \varpi(t)\right] \\
& \leq p+p \varpi(t) K(t)[5 \pi p \varpi(t)] \leq p+p(8 \delta+2)\left[5 \pi p\left(\frac{1}{p} \log (8 \delta+2)\right)^{2}\right],
\end{aligned}
$$

where the first inequality is obtained by using this fact $\left(1+\tan ^{2}(h(t)) \geq \tan (h(t)) \geq 1\right.$ and $t \in(0,1]$. Now, we estimate a lower bound for the value of step size as

$$
\begin{aligned}
\tilde{\alpha}=\frac{1}{\psi^{\prime \prime}(t)} & \geq \frac{1}{p+p(8 \delta+2)\left[5 \pi p\left(\frac{1}{p} \log (8 \delta+2)\right)^{2}\right]} \\
& =\Theta\left(\frac{1}{\delta p^{2}\left(\frac{1}{p} \log (8 \delta+2)\right)^{2}}\right)
\end{aligned}
$$

where the inequality is obtained from the fact that $\Psi(v) \geq p$ and $\delta \geq \sqrt{\frac{p}{2}}$ by Corollary 2.6. These results implies that

$$
f(\tilde{\alpha}) \leq-\frac{\delta^{2}}{\psi^{\prime \prime}(\rho(2 \delta))} \leq \Theta\left(-\frac{\delta}{\log ^{2}(8 \delta+2)}\right) .
$$

This completes the proof of the lemma.

Corollary 4.3. The following results is obtained by using Lemma 4.2 along with the second part of Lemma 2.5.

$$
f(\tilde{\alpha}) \leq \Theta\left(\frac{-\delta}{\log ^{2}(8 \delta+2)}\right) \leq \Theta\left(\frac{-\Psi^{\frac{1}{2}}(v)}{\log ^{2}(\Psi(v))}\right)
$$

\section{ITERATION COMPLEXITY}

In this section, we examine the iteration complexity of Algorithm 1 based on the new kernel function $\psi$ defined by (2.1). During an inner iteration, using $\tilde{\alpha}$ defined by (4.3), as a default value for the step size. Therefore, updating the parameter $\mu$ to $(1-\theta) \mu$, for $\theta \in(0,1)$, implies that $v_{+}=\frac{1}{\sqrt{1-\theta}} v$. Thus, by using Lemma 2.8 along with $\beta=\frac{1}{\sqrt{1-\theta}}$, we imply that

$$
\Psi\left(v_{+}\right) \leq \Psi(v)+\frac{\theta}{2(1-\theta)}(2 \Psi(v)+2 \sqrt{2 n \Psi(v)}+n) .
$$

At the start of each outer iteration of the algorithm and just before updating of the parameter $\mu$, we have $\Psi(v) \leq \tau$. By using (5.1), the value of proximity function $\Psi(v)$ exceeds the threshold $\tau$ after updating of $\mu$. So, the algorithm performs the inner iterations to reduce the value of proximity function such that $\Psi(v) \leq \tau$. To compute the number of inner iterations, we represent the value of proximity function $\Psi(v)$ after $\mu$-update by $\Psi_{0}$, and the subsequent values by $\Psi_{j}$, for $j=1, \ldots, L-1$, where $L$ denotes the total number of inner iterations in an outer iteration. Therefore (5.1) can be rewritten as

$$
\Psi_{0} \leq \tau+\frac{\theta}{2(1-\theta)}(2 \tau+2 \sqrt{2 n \tau}+n)
$$


Our goal in this paper is to work on the large neighborhood of the central path. Thus, we assume that $\tau=O(n) \geq 1$. This fact along with (5.2) implies that $\Psi_{0}=O(n)$. Moreover, in the all inner iterations, we have $\Psi_{j}>\tau \geq 1$. Therefore, from Corollary 4.3, the decrease of $\Psi$ in any inner iteration is then given as

$$
\Psi_{j+1} \leq \Psi_{j}-\kappa \Delta \Psi_{j}, \quad j=0,1, \ldots, L-1,
$$

where $\kappa$ is some positive constant and $\Delta \Psi_{j}$ is defined by

$$
\Delta \Psi_{j}=\frac{\Psi_{j}^{\frac{1}{2}}}{\log ^{2}\left(\Psi_{j}\right)} .
$$

To proceed, we need the following technical lemma.

Lemma 5.1. Given $\alpha \in[0,1]$ and $t \geq-1$, one has $(1+t)^{\alpha} \leq 1+\alpha t$.

Using Lemma 5.1, we can provide the worst case iteration bound for the total number of inner iterations in an outer iteration as follows .

Theorem 5.2. Suppose that $\tau \geq 1$. Then, using (5.3), one has

$$
L \leq 1+\frac{2 \log ^{2}\left(\Psi_{0}\right)}{\kappa} \Psi_{0}^{\frac{1}{2}}
$$

Proof. By using (5.3), for all $j=0,1, \ldots, L-1$, we have

$$
\begin{aligned}
0 \leq \Psi_{j+1}^{\frac{1}{2}} & \leq\left(\Psi_{j}-\frac{\kappa}{\log ^{2}\left(\Psi_{j}\right)} \Psi_{j}^{\frac{1}{2}}\right)^{\frac{1}{2}} \\
& \leq \Psi_{j}^{\frac{1}{2}}\left(1-\frac{\kappa}{2 \log ^{2}\left(\Psi_{j}\right)} \Psi_{j}^{-\frac{1}{2}}\right) \\
& =\Psi_{j}^{\frac{1}{2}}-\frac{\kappa}{2 \log ^{2}\left(\Psi_{j}\right)},
\end{aligned}
$$

where the last inequality is obtained from Lemma 5.1. So, $\Psi_{j+1}^{\frac{1}{2}} \leq \Psi_{0}^{\frac{1}{2}}-\frac{j \kappa}{2 \log ^{2}\left(\Psi_{j}\right)}$. Letting $j=L-1$, we conclude that $0 \leq \Psi_{L}^{\frac{1}{2}} \leq \Psi_{0}^{\frac{1}{2}}-\frac{(L-1) \kappa}{2 \log ^{2}\left(\Psi_{0}\right)}$, which implies that $L \leq 1+\frac{2 \log ^{2}\left(\Psi_{0}\right)}{\kappa} \Psi_{0}^{\frac{1}{2}}$.

For large-update method, we set $\tau=O(n)$ and $\theta=\Theta(1)$. By considering (5.2), we can conclude that $\Psi_{0}=O(n)$. Therefore, we can obtain the following upper bound for the total number of inner iterations in an outer iteration by using Lemma 5.2

$$
L \leq\left\lfloor 1+\frac{2 \log ^{2}\left(\Psi_{0}\right)}{\kappa} \Psi_{0}^{\frac{1}{2}}\right\rfloor=\left\lfloor O\left(\sqrt{n} \log ^{2} n\right)\right\rfloor .
$$

Lemma I.36 of [33] demonstrates that the total number of outer iterations in the large update methods for reaching $n \mu \leq \varepsilon$ is bounded above by $O\left(\frac{1}{\theta} \log \frac{n}{\varepsilon}\right)$. So, we can compute the total number of iterations in Algorithm 1 by multiplying the total number of inner and outer iterations. Hence, the total number of iterations to get an $\varepsilon$ solution for the problems (P) and (D), i.e., a solution that satisfies $x^{T} s=n \mu \leq \varepsilon$, is obtained as follows

$$
O\left(\sqrt{n} \log ^{2} n \log \frac{n}{\varepsilon}\right)
$$


TABLE 2. Considered kernel functions.

\begin{tabular}{|c|c|c|}
\hline$i$ & Kernel functions $\psi_{i}$ & References \\
\hline 1 & $\frac{t^{2}-1}{2}-\int_{1}^{t} \exp \left(\frac{1}{t}-1\right) d x$ & [6] \\
\hline 2 & $\frac{t^{2}-1}{2}-\log (t)$ & [6] \\
\hline 3 & $\frac{t^{2}-1}{2}-\int_{1}^{t} \exp \left(3\left(\tan \left(\frac{\pi}{2+2 x}\right)-1\right)\right) d x$ & [27] \\
\hline 4 & $\frac{t^{2}-1}{2}+\frac{4}{\pi} \cot \left(\frac{\pi t}{1+t}\right)$ & [13] \\
\hline 5 & $\frac{t^{2}-1}{2}+\frac{6}{\pi} \tan \left(\frac{1-t}{2+4 t} \pi\right)$ & [12] \\
\hline 6 & $\frac{t^{2}-1-\log (t)}{2}+\frac{t^{1-q}-1}{2(q-1)}, q=2$ & {$[34]$} \\
\hline 7 & $p \frac{t^{2}-1}{2}+\frac{4}{\pi}\left(\exp \left[p\left(\tan \left(\frac{\pi}{2+2 t}\right)-1\right)\right]-1\right), \quad p \geq 1$ & New kernel function \\
\hline
\end{tabular}

Now, due to [6], this bound yields the so far best known iteration bound for large update methods in terms of trigonometric kernel functions.

\section{NUMERICAL RESULTS}

In this section, some numerical results of performing IPM based on the kernel function on a test problem mentioned in [16] are presented. We implement Algorithm 1 with the new proposed trigonometric kernel function defined by (2.1) along with six existing kernel functions in the literature, which are mentioned in Table 2. We code the algorithm in MATLAB 8.2.0.701 (R2013b) environment and have implemented on a PC with Intel Core i5-7200U CPU and 12GB of RAM memory by double precision format.

Moreover, the value of step size in the inner iterations of all experiments is calculated as an approximate value of the default step size in the related references. For the mentioned test problem in [16], we have $n=2 m$, and for all $1 \leq i \leq m$, the parameters of the problem are given by

$$
A=\left[I_{m}, I_{m}\right], \quad b=2 e_{m} \quad c=\left[-e_{m} ; 0_{m}\right],
$$

where the identity matrix of size $m \times m$ is denoted by $I_{m}$, and $0_{m}$ and $e_{m}$ are the zero vector and the all-one vector of length $m$, respectively. We choose the strictly feasible initial point as

$$
x^{0}=\left[e_{m} ; e_{m}\right], \quad s^{0}=\left[e_{m} ; 2 e_{m}\right], \quad y^{0}=-2 e_{m} .
$$

We set the parameters of Algorithm 1 as below

$$
\mu^{0}=1, \quad \varepsilon=10^{-8}, \quad \tau=3, \quad \theta \in\{0.95,0.99\} .
$$

Moreover, we choose the paramors $m \in\{375,750,1500,3000,7500\}$ and $p \in\{1,2,3\}$ in the setting of the new proposed kernel function. The values of $p$ are considered according to the fact that the algorithms obtain the best iteration complexity when $p=O(\log n)$. We present the total number of inner iterations of performing Algorithm 1 based on the kernel functions defined in Table 1 and new proposed kernel function in Tables 3 and 4. In these tables, "Iter", "Time" and "gap" denote the number of iterations, CPU time (in second) and the value of $c^{T} x-b^{T} y$, respectively. We also use $\psi_{7, p}$ for denoting the new proposed kernel function with different values $p$. Results in Tables 3 and 4 show that the new proposed kernel function outperforms the other considered kernel functions. 
TABLE 3. Numerical results of performing Algorithm 1 with $\theta=0.95$.

\begin{tabular}{|c|c|c|c|c|c|c|}
\hline$\psi_{i}$ & & & & $m$ & & \\
\hline & & 375 & 750 & 1500 & 3000 & 7500 \\
\hline & Iter & 200 & 266 & 315 & 339 & 423 \\
\hline \multirow[t]{3}{*}{$\psi_{1}$} & Time & 24.38 & 89.42 & 368.64 & 2168.76 & 14537.45 \\
\hline & gap & $8.42 \mathrm{E}-09$ & $1.62 \mathrm{E}-08$ & $3.12 \mathrm{E}-09$ & $5.62 \mathrm{E}-09$ & 2.91E-09 \\
\hline & Iter & 189 & 257 & 304 & 326 & 436 \\
\hline \multirow[t]{3}{*}{$\psi_{2}$} & Time & 24.16 & 85.80 & 325.76 & 1984.98 & 14872.87 \\
\hline & gap & $8.25 \mathrm{E}-09$ & 2.98E-08 & 3.09E-09 & $6.41 \mathrm{E}-09$ & 7.81E-09 \\
\hline & Iter & 235 & 298 & 312 & 324 & 418 \\
\hline \multirow[t]{3}{*}{$\psi_{3}$} & Time & 37.14 & 108.54 & 341.39 & 1973.87 & 13985.65 \\
\hline & gap & 8.19E-09 & $1.65 \mathrm{E}-08$ & $1.67 \mathrm{E}-08$ & $3.62 \mathrm{E}-09$ & $8.48 \mathrm{E}-09$ \\
\hline & Iter & 176 & 223 & 283 & 319 & 397 \\
\hline \multirow[t]{3}{*}{$\psi_{4}$} & Time & 19.81 & 72.82 & 327.71 & 1832.61 & 13565.71 \\
\hline & gap & 8.34E-09 & $1.96 \mathrm{E}-08$ & $1.02 \mathrm{E}-08$ & 2.05E-09 & $5.12 \mathrm{E}-09$ \\
\hline & Iter & 194 & 261 & 308 & 345 & 461 \\
\hline \multirow[t]{3}{*}{$\psi_{5}$} & Time & 20.47 & 79.81 & 359.62 & 2171.01 & 14891.38 \\
\hline & gap & 8.37E-09 & $1.61 \mathrm{E}-08$ & $2.67 \mathrm{E}-09$ & 4.35E-09 & 8.19E-09 \\
\hline & Iter & 189 & 272 & 319 & 342 & 439 \\
\hline \multirow[t]{3}{*}{$\psi_{6}$} & Time & 22.94 & 81.37 & 381.06 & 2153.87 & 14624.43 \\
\hline & gap & $2.15 \mathrm{E}-08$ & $1.51 \mathrm{E}-08$ & 2.74E-09 & 4.29E-09 & 8.01E-09 \\
\hline & Iter & 157 & 193 & 220 & 291 & 353 \\
\hline \multirow[t]{3}{*}{$\psi_{7,1}$} & Time & 16.12 & 60.25 & 230.43 & 1485.16 & 9831.45 \\
\hline & gap & 7.79E-09 & $2.12 \mathrm{E}-08$ & 7.18E-09 & 6.19E-09 & $8.35 \mathrm{E}-09$ \\
\hline & Iter & 157 & 189 & 220 & 289 & 342 \\
\hline \multirow[t]{3}{*}{$\psi_{7,2}$} & Time & 17.12 & 65.47 & 231.19 & 1581.00 & 10243.10 \\
\hline & gap & $1.03 \mathrm{E}-08$ & $1.97 \mathrm{E}-08$ & $1.32 \mathrm{E}-08$ & $6.28 \mathrm{E}-09$ & 7.32E-09 \\
\hline & Iter & 161 & 188 & 248 & 297 & 328 \\
\hline \multirow[t]{2}{*}{$\psi_{7,3}$} & Time & 18.34 & 66.25 & 278.01 & 1531.19 & 10.423 .00 \\
\hline & gap & $9.41 \mathrm{E}-09$ & 8.31E-09 & $1.01 \mathrm{E}-08$ & 4.64E-09 & 8.32E-09 \\
\hline
\end{tabular}

\section{THE CONCLUSION}

In this paper, we introduced an interior point algorithm for the LO problem based on a new trigonometric kernel function. By using some simple analysis tools and the e-convexity property of the kernel function that plays an important role in deriving a default value for the step size, we computed the worst case iteration complexity analysis for the large update method. We showed that IPM based on the new proposed kernel function has the best known iteration complexity bound for large-update methods, i.e., $O\left(\sqrt{n} \log n \log \frac{n}{\varepsilon}\right)$. Finally, we performed Algorithm 1 based on the new proposed kernel function and 
TABLE 4. Numerical results of performing Algorithm 1 with $\theta=0.99$.

\begin{tabular}{|c|c|c|c|c|c|c|}
\hline \multirow[t]{2}{*}{$\psi_{i}$} & \multicolumn{6}{|c|}{$m$} \\
\hline & & 375 & 750 & 1500 & 3000 & 7500 \\
\hline & Iter & 182 & 237 & 263 & 282 & 359 \\
\hline \multirow[t]{3}{*}{$\psi_{1}$} & Time & 24.09 & 88.08 & 324.04 & 1763.23 & 11452.41 \\
\hline & gap & 1.64E-09 & 3.19E-09 & 6.19E-09 & $1.22 \mathrm{E}-08$ & 2.17E-09 \\
\hline & Iter & 173 & 245 & 281 & 302 & 381 \\
\hline \multirow[t]{3}{*}{$\psi_{2}$} & Time & 23.25 & 80.35 & 302.14 & 1691.41 & 11191.40 \\
\hline & gap & $1.51 \mathrm{E}-09$ & $3.14 \mathrm{E}-09$ & $6.01 \mathrm{E}-09$ & $1.26 \mathrm{E}-08$ & 2.43E-09 \\
\hline & Iter & 221 & 251 & 288 & 307 & 373 \\
\hline \multirow[t]{3}{*}{$\psi_{3}$} & Time & 32.14 & 103.07 & 338.97 & 1837.98 & 12342.28 \\
\hline & gap & $1.45 \mathrm{E}-09$ & 2.99E-09 & 6.71E-09 & $1.67 \mathrm{E}-08$ & 2.01E-09 \\
\hline & Iter & 151 & 209 & 246 & 271 & 325 \\
\hline \multirow[t]{3}{*}{$\psi_{4}$} & Time & 19.34 & 79.31 & 302.17 & 1692.68 & 11109.14 \\
\hline & gap & 1.44E-09 & 3.07E-09 & $6.43 \mathrm{E}-09$ & $1.81 \mathrm{E}-08$ & 2.71E-09 \\
\hline & Iter & 171 & 231 & 266 & 315 & 383 \\
\hline \multirow[t]{3}{*}{$\psi_{5}$} & Time & 19.01 & 81.12 & 312.72 & 1701.01 & 11601.54 \\
\hline & gap & 1.39E-09 & 2.97E-09 & $6.22 \mathrm{E}-09$ & $1.27 \mathrm{E}-08$ & 4.91E-010 \\
\hline & Iter & 156 & 191 & 250 & 292 & 347 \\
\hline \multirow[t]{3}{*}{$\psi_{6}$} & Time & 22.07 & 85.12 & 319.45 & 1757.16 & 11910.15 \\
\hline & gap & $1.44 \mathrm{E}-09$ & 3.09E-09 & $6.43 \mathrm{E}-09$ & $1.89 \mathrm{E}-08$ & $4.32 \mathrm{E}-010$ \\
\hline & Iter & 138 & 172 & 202 & 240 & 279 \\
\hline \multirow[t]{3}{*}{$\psi_{7,1}$} & Time & 16.08 & 57.37 & 253.59 & 1329.00 & 8197.52 \\
\hline & gap & 4.76E-09 & $2.54 \mathrm{E}-09$ & $1.16 \mathrm{E}-08$ & 4.87E-09 & 2.76E-09 \\
\hline & Iter & 140 & 173 & 209 & 231 & 270 \\
\hline \multirow[t]{3}{*}{$\psi_{7,2}$} & Time & 17.12 & 58.25 & 262.16 & 1341.71 & 8221.10 \\
\hline & gap & $6.09 \mathrm{E}-09$ & $1.12 \mathrm{E}-08$ & 3.32E-09 & $5.18 \mathrm{E}-09$ & $1.06 \mathrm{E}-08$ \\
\hline & Iter & 140 & 179 & 223 & 249 & 279 \\
\hline \multirow[t]{2}{*}{$\psi_{7,3}$} & Time & 17.25 & 62.13 & 271.14 & 1402.98 & 8315.00 \\
\hline & gap & $1.34 \mathrm{E}-09$ & $3.00 \mathrm{E}-09$ & $6.41 \mathrm{E}-09$ & $1.50 \mathrm{E}-08$ & $3.01 \mathrm{E}-09$ \\
\hline
\end{tabular}

some existing kernel functions in the literature. Numerical results demonstrated that the new proposed kernel function has better results than the other kernel functions.

\section{REFERENCES}

[1] N.K. Karmarkar, A new polynomial-time algorithm for linear programming, Combinatorica, 4 (1984), 373-395.

[2] M. Kojima, S. Mizuno, A. Yoshise, A primal-dual interior point algorithm for linear programming, in: N. Megiddo (Ed.), Progress in Mathematical Programming Interior-Point and Related Methods, pp. 29-47, Springer Verlag, New York, 1989. 
[3] N. Megiddo, Pathways to the optimal set in linear programming, in: N. Megiddo (Ed.), Progress in Mathematical Programming Interior-Point and Related Methods, pp. 131-158, Springer-Verlag, New York, 1989.

[4] Y.E. Nesterov, A.S. Nemirovskii, Interior Point Polynomial Algorithms in Convex Programming, SIAM Studies in Applied Mathematics, Vol.13, SIAM, Philadelphia, PA, 1994.

[5] J. Peng, C. Roos, T. Terlaky, A new class of polynomial primal-dual methods for linear and semidefinite optimization, Eur. J. Oper. Res. 143 (2002), 234-256.

[6] Y.Q. Bai, M. El Ghami, C. Roos, A comparative study of kernel functions for primal-dual interior-point algorithms in linear optimization, SIAM J. Optim. 15 (2004), 101-128.

[7] Y.Q. Bai, J.L. Guo, C. Roos, A new kernel function yielding the best known iteration bounds for primal-dual interior-point algorithms, Acta Math. Sin. Engl. Ser. 25 (2009), 2169-2178.

[8] Y.Q. Bai, G. Lesaja, C. Roos, G.Q. Wang, M. El Ghami, A class of large-update and small-update primal-dual interiorpoint algorithms for linear optimization, J. Optim. Theory Appl. 138 (2008), 341-359.

[9] M. El Ghami, C. Roos, Generic primal-dual interior point methods based on a new kernel function, RAIRO-Oper. Res. 42 (2008), 199-213.

[10] J. Peng, C. Roos, T. Terlaky, Self-Regularity: A New Paradigm for Primal-Dual Interior-Point Algorithms, Princeton University Press, Princeton, (2002).

[11] M. Reza Peyghami, K. Amini, A kernel function based interior-point methods for solving $P_{*}(\kappa)$-linear complementarity problem, Acta Math. Sin. Engl. Ser. 26 (2010), 1761-1778.

[12] M. El Ghami, Z.A. Guennoun, S. Boula, T. Steihaug, Interior-point methods for linear optimization based on a kernel function with a trigonometric barrier term, J. Comput. Appl. Math. 236 (2012), 3613-3623.

[13] B. Kheirfam, Primal-dual interior-point algorithm for semidefinite optimization based on a new kernel function with trigonometric barrier term, Numer. Algo. 61 (2012), 659-680.

[14] M.R. Peyghami, S. Fathi-Hafshejani, L. Shirvani, Complexity of interior-point methods for linear optimization based on a new trigonometric kernel function, J. Comput. Appl. Math. 255 (2014), 74-85.

[15] M. El Ghami, Primal-dual algorithms for $P_{*}(\kappa)$-linear complementarity problems based on kernel-function with trigonometric barrier term, In: Migdalas A., Sifaleras A., Georgiadis C., Papathanasiou J., Stiakakis E. (eds) Optimization Theory, Decision Making, and Operations Research Applications, Springer Proceedings in Mathematics \& Statistics, vol. 31, pp. 331-349, Springer, New York, NY, 2013.

[16] M. Bouafia, D. Benterki, A. Yassine, An efficient primal-dual interior point method for linear programming problems based on a new kernel function with a trigonometric barrier term, J. Optim. Theory Appl. 170 (2016), 528-545.

[17] X. Wang, X. Ou, T. Zhang, J. Chen, An alternate minimization method beyond positive definite proximal regularization: convergence and complexity, J. Nonlinear Var. Anal. 3 (2019), 333-355.

[18] S. Fathi-Hafshejani, M. Fatemi, M.R. Peyghami, An interior-point method for $P_{*}(\kappa)$-linear complementarity problem based on a trigonometric kernel function, J. Appl. Math. Comput. 48 (2015), 111-128.

[19] S. Fathi-Hafshejani, H. Mansouri, M.R. Peyghami, A large-update primal-dual interior-point algorithm for second-order cone optimization based on a new proximity function, Optimization, 65 (2016), 1477-1496.

[20] S. Fathi-Hafshejani, A. Fakharzadeh J., M.R. Peyghami, A unified complexity analysis of interior point methods for semidefinite problems based on trigonometric kernel functions, Optimization, 67 (2018), 113-137.

[21] M.R. Peyghami, S. Fathi-Hafshejani, An interior point algorithm for solving convex quadratic semidefinite optimization problems using a new kernel function, Iranian J. Math. Sci. Info. 12 (2017), 131-152.

[22] M.R. Peyghami, S. Fathi-Hafshejani, S. Chen, A prima dual interior-point method for semidefinite optimization based on a class of trigonometric barrier functions, Oper. Res. Lett. 44 (2016), 319-323.

[23] M. Bouafia, A. Yassine, An efficient twice parameterized trigonometric kernel function for linear optimization, Optim. Engineering, (2019), doi.org/10.1007/s11081-019-09467-w.

[24] S. Fathi Hafshejani, A. Fakharzadeh J., An interior point method for $P_{*}(\kappa)$-horizontal linear complementarity problem based on a new proximity function, J. Appl. Math. Comput. (2019), doi.org/10.1007/s12190-019-01284-9.

[25] S. Fathi-Hafshejani, A. Fakharzadeh J., M.R. Peyghami, S. Chen, Complexity of interior point methods for a class of linear complementarity problems using a kernel function with trigonometric growth term, J. Optim. Theory Appl. 178 (2018), 935-949. 
[26] S. Fathi-Hafshejani, M.R. Peyghami, A. Fakharzadeh J., An efficient primal-dual interior point method for linear programming problems based on a new kernel function with a finite exponential-trigonometric barrier term, Optim. Engineering, (2019), doi.org/10.1007/s11081-019-09436-3.

[27] M.R. Peyghami, S. Fathi-Hafshejani, Complexity analysis of an interior point algorithm for linear optimization based on a new poriximity function, Numer. Algo. 67 (2014), 33-48.

[28] X.Z. Cai, G.Q. Wang, M. El Ghami, Y.J. Yue, Complexity analysis of primal-dual interior-point methods for linear optimization based on a new parametric kernel function with a trigonometric barrier term, Abst. Appl. Anal. 2014 (2014), Art. ID 710158.

[29] B. Kheirfam, M. Moslem, A polynomial-time algorithm for linear optimization based on a new kernel function with trigonometric barrier term, Yugoslav J. Oper. Res. 25 (2015), 233-250.

[30] X. Li, M. Zhang, Interior-point algorithm for linear optimization based on a new trigonometric kernel function, Oper. Res. Lett. 43 (2015), 471-475.

[31] R.D.C. Monteiro, I. Adler, Interior-point path following primal-dual algorithms: Part I: linear programming, Math. Program. 44 (1989), 27-41.

[32] G. Sonnevend, An analytic center for polyhedrons and new classes of global algorithms for linear (smooth, convex) programming, in: A. Prakopa, J. Szelezsan and B. Strazicky (Eds.), Lecture Notes in Control and Information Sciences, Vol.84, pp.866-876, Springer-Verlag, Berlin, 1986.

[33] C. Roos, T. Terlaky, J-Ph. Vial, Theory and Algorithms for Linear Optimization: An Interior Point Approach, Springer, New York, 2005.

[34] M. Bouafia, D. Benterki, A. Yassine, An efficient parameterized logarithmic kernel function for linear optimization, Optim. Lett. 12 (2018), 1079-1097. 